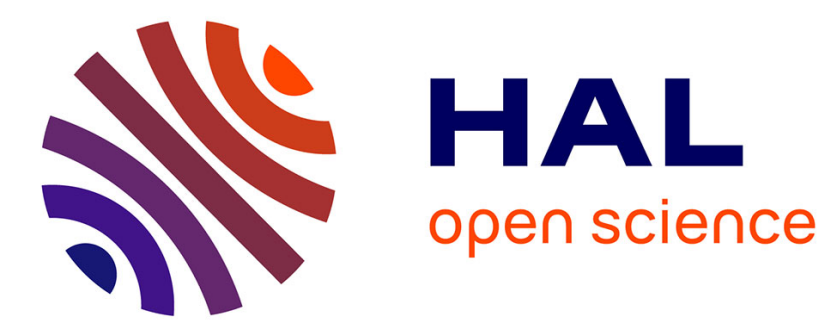

\title{
L'Institut littéraire Gorki à Moscou : la patrimonialisation de la création littéraire
}

Sylvia Chassaing

\section{To cite this version:}

Sylvia Chassaing. L'Institut littéraire Gorki à Moscou: la patrimonialisation de la création littéraire.

Culture et Musées, 2021, 38, pp.51 - 68. 10.4000/culturemusees.6673 . hal-03471476

\author{
HAL Id: hal-03471476 \\ https://hal.science/hal-03471476
}

Submitted on 8 Dec 2021

HAL is a multi-disciplinary open access archive for the deposit and dissemination of scientific research documents, whether they are published or not. The documents may come from teaching and research institutions in France or abroad, or from public or private research centers.
L'archive ouverte pluridisciplinaire HAL, est destinée au dépôt et à la diffusion de documents scientifiques de niveau recherche, publiés ou non, émanant des établissements d'enseignement et de recherche français ou étrangers, des laboratoires publics ou privés. 


\section{Culture \& Musées}

Muséologie et recherches sur la culture

$38 \mid 2021$

Patrimonialisations de la littérature

Dossier

\section{L'Institut littéraire Gorki à Moscou : la patrimonialisation de la création littéraire}

The Gorky Literature Institute in Moscow: from patrimonialisation to literary creation

El Instituto Literario Gorki de Moscú: la patrimonialización de la creación literaria

\section{Sylvia ChASSAING}

p. 51-68

https://doi.org/10.4000/culturemusees.6673

\section{Résumés}

Français English Español

Fondé en 1932 à l'initiative de Staline et avec l'aval de Maxim Gorki, l'Institut littéraire Gorki (Litinstitut) a pour but de former les futurs écrivains de la jeune Union soviétique. Initialement vu comme un moyen de canaliser et de contrôler les vocations d'écrivains, l'Institut devient très vite une machine complexe visant à patrimonialiser " par avance » la littérature soviétique, alors que celle-ci est, dans les années 1930, tout juste naissante. Cela passe d'abord par l'enseignement de l'écriture littéraire. Très rapidement, l'Institut se dote en plus d'une bibliothèque de dépôt et d'un musée portant sur Maxim Gorki. On y crée, enfin, un laboratoire de recherche dont le but est d'établir une histoire marxiste-léniniste de la littérature de tous les pays et de toutes les époques. Sur le modèle industriel soviétique, l'Institut Gorki en vient donc à prendre en charge l'ensemble des étapes de la production littéraire : les contemporains de sa fondation ont ainsi pu parler à son propos d'un « combinat » littéraire (literaturnyj kombinat).

À partir des statuts de création de l'université, des ouvrages de recherche produits en son sein et des quatre tomes de Souvenirs du Litinstitut publiés par les presses de l'établissement, on montre que l'intérêt du Litinstitut pour comprendre ce qui se joue dans les mécanismes de patrimonialisation est son caractère ouvertement tourné vers l'avenir, qui montre que la patrimonialisation, y compris de la littérature, est un processus de création (d'une "identité » collective, d'une " communauté imaginaire » appuyée sur une définition singulière de la littérature) autant qu'un processus de conservation.

Founded in 1933 at the initiative of Stalin and Maxim Gorky, the Gorky Literature Institute (Litinstitut) was intended to train the future authors of the nascent Soviet Union. Initially seen as a means of channeling and controlling authors' vocations, the Institute quickly became a complex machine aimed at creating heritage out of soviet literature "from the outset" despite this genre being at its very beginnings in the 1930s. This was accomplished firstly through instruction in 
literary writing. The Institute was quickly made the home of a legal deposit library and a museum dedicated to Maxim Gorky. Finally, a research lab was created in order to establish a MarxistLeninist history of literature that would span all countries and periods. Following the Soviet industrial model, the Gorky Literature Institute ended up covering all the steps in the process of literary creation: contemporaries referred to it as a "literary kombinat" (literaturnyj kombinat).

From the statutes of the university, the research texts that it published and the four volumes of the Litinstitut's Souvenirs, it is made apparent that the Litinstitut's concern for understanding what happens in the mechanisms of creating heritage is its openly forward-looking nature, showing that this process, including its application to literature, is a creative one (of a collective "identity", of an "imagined community" based on a singular definition of literature) as much as a process of preservation.

El Instituto Literario Gorki (Litinstitut), fundado en 1932 por iniciativa de Stalin y con la aprobación de Maxim Gorki, tiene como objetivo formar a los futuros escritores de la joven Unión Soviética. Considerado inicialmente como un medio para canalizar y controlar las vocaciones de los escritores, el Instituto se convirtió muy pronto en una compleja máquina que pretendía patrimonializar anticipadamente, la literatura soviética, la cual, en los años treinta, a penas se encontraba naciendo. La primera etapa consiste en enseñar la escritura literaria. Rápidamente, el Instituto adquiere también una biblioteca depositaria y un museo sobre Maxim Gorky. Por último, se crea un laboratorio de investigación con el objetivo de establecer una historia marxista-leninista de la literatura de todos los países y épocas. Basado en el modelo industrial soviético, el Instituto Gorki llega a hacerse cargo de todas las etapas de la producción literaria: los contemporáneos de su fundación pudieron hablar de él como de un "combinat" literario (literaturnyj kombinat).

A partir de los estatutos fundacionales de la universidad, de los trabajos de investigación producidos y de los cuatros tomos de Souvenirs del Litinstitut publicadas por la prensa del establecimiento, mostramos que el interés del Litinstitut por entender lo que está en juego en los mecanismos de patrimonialización, es su carácter abiertamente prospectivo. Esto demuestra que el patrimonio, incluyendo la literatura, es un proceso de creación (de una "identidad" colectiva, de una "comunidad imaginaria" basada en una definición singular de la literatura) tanto como un proceso de conservación.

\section{Entrées d'index}

Mots-clés : URSS, création littéraire, histoire littéraire, institution, construction nationale

Keywords: USSR, literary creation, literary history, institution, national construction

Palabras clave: URSS, creación literaria, historia literaria, institución, construcción nacional

\section{Notes de la rédaction}

Manuscrit reçu le 26 janvier 2021

Version révisée reçue le 10 juin 2021

Article accepté pour publication le 26 juin 2021

\section{Texte intégral}

Fondé en 1932 à l'initiative de Staline, l'Institut littéraire Gorki (Litinstitut) a pour but de former les futurs écrivains de la jeune Union soviétique. Initialement vu comme un moyen de canaliser et de contrôler les vocations d'écrivains - qui naissent de façon spontanée et se multiplient à travers le pays dans les clubs et les cercles littéraires ouvriers (Dobrenko, 1999 : 420) -, l'Institut devient très vite une machine complexe visant à patrimonialiser " par avance » la littérature soviétique, alors que celle-ci est, dans les années 1930, tout juste naissante. Sa création a lieu pendant la " grande retraite " stalinienne qui voit le retour, après l'effervescence de la période révolutionnaire, du conservatisme esthétique et du nationalisme (Timasheff, 1946), et elle s'inscrit dans une mise au pas de la production littéraire dont participent également la création de l'Union des écrivains en 1934, ainsi que l'affirmation de la doctrine du réalisme socialiste à l'occasion du premier congrès de cette Union. La création de l'Institut Gorki est donc un des aspects d'un processus plus large de contrôle de la production littéraire, qui passe par une patrimonialisation des ouvres passées et présentes en fonction d'un canon prédéfini par l'État.

2 La révolution russe, dans la réflexion que ses initiateurs ont menée sur le patrimoine, ne peut pas ne pas évoquer la Révolution française. Comme le remarque Julie Deschepper (2018 : 81), " ces deux révolutions patrimoniales entraînèrent des 
comportements similaires : vandalisme [...], iconoclasme, sémioclasme, confiscation, aussi bien que volonté de préserver le patrimoine ». La différence fondamentale entre elles réside dans les régimes d'historicité qui sont les leurs : si la Révolution française, comme l'affirme Dominique Poulot (2006 : 72), élit son patrimoine au nom d'une " réhabilitation du vrai », intemporel et toujours présent, la révolution russe, tournée vers un avenir qu'elle est, à terme, censée incarner, crée le patrimoine de l'avenir en même temps qu'elle conserve et réinterprète celui du passé.

3 L'Institut littéraire Gorki a le mérite de concentrer ces deux aspects de la politique patrimoniale soviétique, conservation et réinterprétation du patrimoine passé d'une part, création du patrimoine présent et à venir d'autre part, ce qui est une manière d'assurer le lien entre passé prérévolutionnaire et présent soviétique. Les œuvres déjà canonisées (enseignées et reconnues avant la révolution) sont réinterprétées pour s'inscrire dans un nouveau canon, soviétique, dont les enjeux sont différents et que l'Institut Gorki permet de certifier. C'est ce canon, réinterprété, qui permet de présenter la littérature soviétique contemporaine comme un patrimoine littéraire en construction. L'étude de cette institution permet donc de comprendre que ces deux aspects sont en réalité indissociables : la réinterprétation du patrimoine passé est mise au service de la création du patrimoine présent, qui le légitime en retour. À travers l'Institut Gorki, l'Union soviétique se réapproprie donc le patrimoine littéraire russe antérieur en l'intégrant à son canon et, par ailleurs, elle place la création littéraire soviétique naissante dans sa continuité, au prix d'une réinterprétation des œuvres du passé : il est bien une forme de "pratique symbolique de construction d'un rapport au passé " (Davallon, 2006 : 193) dans lequel le passé vient justifier le régime politique en place et ses principes politiques.

$4 \quad$ Notre but, dans cet article, est de montrer quels principes ont présidé à la fondation de l'Institut Gorki, de quelle façon celui-ci établit un patrimoine littéraire soviétique, de quelle représentation de la littérature contemporaine et de son lien au passé il est vecteur, et comment ces représentations se manifestent dans son organisation et dans son maintien au fil des décennies, même après la chute de l'Union soviétique. Nous verrons d'abord dans quel contexte naît l'Institut Gorki. Cela permettra, dans un deuxième temps, de décrire et de comprendre son fonctionnement et les valeurs qu'il reflète. Nous envisagerons, enfin, l'héritage contemporain de cette patrimonialisation sur le vif de la littérature soviétique.

\section{Encadrer la littérature soviétique naissante}

L'Institut Gorki, qui se donne pour but de former les écrivains du nouveau régime, constitue une nouveauté par rapport aux formes qu'a pu prendre l'apprentissage littéraire, aussi bien en Russie qu'en Europe occidentale. Dans la Russie du XIXe siècle, comme en France, les cercles littéraires, cénacles et autres regroupements informels, où les textes des jeunes auteurs circulent et sont discutés, jouent un rôle de formation pour les écrivains débutants, mais ils sont, par définition, dépourvus de toute autorité institutionnelle. L'Institut Gorki a pour originalité d'allier à la visée formatrice qui est initialement la sienne une fonction de consécration qui passe par l'étude et la conservation de la littérature du passé mais aussi du présent.

\section{Contexte de création : cercles et clubs littéraires ouvriers}

6 Dès le tout début du siècle, le poète symboliste Brioussov projette de fonder une " école de poésie ». Lors de conférences en 1918, il précise qu'elle aura pour fonction d'enseigner " le métier [remeslo] du poète », mais en insistant sur le fait qu'il ne serait pas possible de «produire » (sozdavat') des poètes sans dispositions préexistantes, 
donc sans une forme de "génie » (Kurilov, $2011: 260-261)$. Des écrivains plus âgés et plus expérimentés y viendraient en aide aux plus jeunes pour leur montrer les ficelles du métier.

7 Après la révolution, un Institut littéraire et artistique supérieur (Vysšij literaturnohudožestvennyj Institut) voit le jour à Moscou en 1919, avant d'être déplacé à SaintPétersbourg et d'être, de fait, dissout dans le département de littérature de l'université de cette ville. La formation des écrivains y incluait, entre autres, des cours d'histoire littéraire fondés, à en juger par leurs intitulés (" La littérature avant Gogol », " La littérature après Gogol »), sur une vision de l'histoire littéraire comme panthéon de figures exemplaires, et qui organisent l'histoire de la littérature non pas autour de notions, de courants littéraires, mais de personnalités marquantes (Kurilov, 2015 : s. p.).

8 Parallèlement, au lendemain de la révolution, mais aussi dès avant elle, de nombreux clubs littéraires ouvriers apparaissent de façon spontanée. Le directeur du club, la plupart du temps un écrivain déjà publié, y joue le rôle de guide ou de professeur pour les jeunes poètes et romanciers (Dobrenko, 1999 : 420). Les manuels d'écriture se multiplient (ibid. : 385). Les revues littéraires, qui foisonnent, comptent enfin parmi leurs comités de rédaction des consultants chargés d'évaluer les textes qui leur sont soumis et, surtout, de guider et de conseiller leurs auteurs (ibid. : 415-416) ${ }^{1}$.

On voit donc apparaître deux conceptions opposées de la littérature et de sa production. D’une part, les cercles littéraires, souvent ouvriers, entretiennent l'idée d'un artisanat littéraire et, indirectement, la conception d'un écrivain travailleur dont l'apprentissage se ferait par compagnonnage, indépendamment d'une " culture » qui est parfois congédiée car " bourgeoise ». D’autre part, en contexte de contrôle idéologique accru, ce qui est le cas au début des années 1930, le caractère spécifique de " l'artisanat littéraire » rend ce fonctionnement suspect : il faut fournir un contenu adéquat à la nouvelle littérature soviétique. Reste à savoir quel contenu il faut transmettre à la jeune génération littéraire soviétique.

\section{Les débuts de l'Institut Gorki : une panthéonisation sur le vif}

10 La fondation de l'Institut Gorki se présente comme une réponse institutionnelle à cette contradiction, d'une part entre la démocratisation de la pratique littéraire et son assimilation à un artisanat, à un savoir-faire ouvrier, d'autre part avec la nécessité croissante pour le Parti et l'État d'en contrôler le contenu. Comme ailleurs dans la vie politique soviétique, cet encadrement passe par l'assignation d'une figure tutélaire : c'était Lénine pour l'idéologie, ce sera Gorki pour la littérature. Le geste même de fondation de l'Institut Gorki est donc celui d'une panthéonisation, de son vivant, de Gorki, censé incarner la littérature soviétique (Dobrenko, 1999 : 429). Cette panthéonisation s'inscrit dans une culture héroïque naissante dans les années 1930 et encouragée par Staline, qui érige une série de modèles dont les représentations visent à évoquer l'immortalité et la perfection (Unfried, 1999 : s. p.) - la figure la plus connue à cet égard étant celle de Stakhanov. Le choix de Gorki, dont le positionnement par rapport au Parti communiste est loin d'être exemplaire et qui a passé la majeure partie des années 1920 à l'étranger, s'explique par la proximité entre ce panthéon naissant de héros soviétique et l'œuvre même de Gorki, dans laquelle le mythe du héros du peuple prend une place croissante (Günther, 1993). Nommer l'institut d'après Gorki peut alors être compris aussi bien comme une volonté de rendre hommage à l'écrivain que comme un moyen de donner ses héros et la prose qui les décrit en modèle aux jeunes générations de littérateurs.

11 En effet, Gorki lui-même a pris part à l'intérêt suscité par l'idée d'une éducation des ouvriers et des masses en général à la création littéraire. Gorki en caresse le projet depuis longtemps et en a fait part à Lénine dans les années 1920 (Kurilov, 2011 : 260). Celui-ci estime toutefois que l'on manque de cadres et qu'il faut attendre que la révolution ait fait émerger une nouvelle littérature avant de pouvoir l'enseigner, ce qui 
ressemble plutôt à une réponse dilatoire pour éviter de s'en occuper et n'est pas très cohérent avec d'autres positions de Lénine sur la littérature (ibid. : 262). Gorki poursuit néanmoins sa réflexion dans la revue qu'il dirige, intitulée L'Apprentissage littéraire (Literaturnaâ Učëba).

Les clubs littéraires ouvriers se multipliant, l'idée d'un lieu d'apprentissage pour les jeunes écrivains réapparaît avec force au début des années 1930 : Staline relance le mouvement pour la création d'un institut de formation littéraire en 1932. Dans une lettre à Gorki, il écrit : "Ne faudrait-il pas, à votre avis, construire une université littéraire en votre nom dans le club des littérateurs ou à côté de lui ?² » (ibid. : 275). Au milieu de la floraison de clubs littéraires ouvriers, Staline envisage l'institutionnalisation de ces rassemblements spontanés sous la forme d'un établissement d'études supérieures (vuz), qui est amené à remplacer le plus informel et plus horizontal « club des littérateurs ».

13 La date même de la fondation de l'Institut est révélatrice de la volonté de faire de Gorki le père fondateur de la jeune littérature soviétique. En effet, même si les inscriptions ne commencent que l'année suivante, en 1933, l'Institut voit officiellement le jour le 17 septembre 1932, date choisie pour honorer les quarante ans d'activité littéraire de Gorki et son investissement dans la formation des écrivains ouvriers et paysans. L'Institut doit également recueillir l'ensemble de sa documentation, ainsi que contenir un musée portant sur sa production et sa vie. Deux ans plus tard, Gorki est nommé à la tête de l'Union des écrivains soviétiques.

La fondation de l'Institut Gorki s'inscrit bien dans un mouvement plus large d'institutionnalisation et de contrôle étatique accru de pratiques littéraires jusqu'alors plus spontanées, et ce processus se fait sous la tutelle symbolique d'un écrivain qui acquiert un statut de patrimoine vivant et dont les œuvres font désormais partie du canon littéraire.

\section{Créer et transmettre un patrimoine littéraire}

Outre son statut d'initiateur et d'ancêtre de la littérature soviétique, Gorki joue également un rôle dans l'établissement du programme du Litinstitut, notamment en influant très fortement sur le contenu des enseignements, selon la conception de la création littéraire qu'il a développée auparavant dans nombre de ses écrits. Refusant des propositions visant à inclure une formation en sciences exactes, Gorki insiste en outre sur l'importance d'une connaissance extensive de l'histoire littéraire, accompagnée de notions en philosophie et en philologie.

Cela explique que, dans les statuts de 1932, le Litinstitut se voit assigner deux tâches :

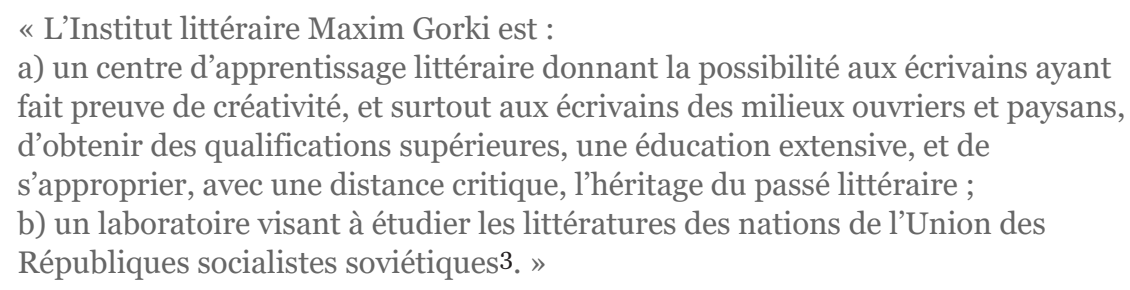

Dans ces statuts, ainsi que dans les autres textes officiels qui accompagnent la fondation de l'Institut, la place du patrimoine littéraire est d'emblée conçue comme centrale pour la formation des futurs écrivains : "l'héritage du passé littéraire » (nasledie literaturnogo prošlogo) est défini comme l'objet d'étude principal des futurs écrivains, même si sa mention vient avec une réserve, celle d'une nécessaire « distance critique » liée aux divergences idéologiques entre la littérature soviétique à venir et celle des époques précédentes : l'objet de l'étude, le patrimoine littéraire à conserver et à étudier pour les nouveaux écrivains, n'est donc pas le corpus littéraire en lui-même, mais l'interprétation qui en est faite, à savoir sa relecture communiste qui doit apporter une confirmation concernant les formes et l'issue de la lutte des classes jusqu'à la révolution de 1917. 
Par ailleurs, contrairement aux projets initiaux qui n'envisageaient l'institution que comme un lieu de formation pratique pour écrivains, ces premiers statuts dotent également l'Institut d'une ambition scientifique. L'Institut est en effet un « laboratoire d'étude des littératures des nations d'URSS ». L'assimilation de la littérature et de son étude à une science exacte est dans l'air du temps, mais l'ambiguïté du mot « laboratoire » est ici significative d'une volonté à la fois de produire et d'étudier la littérature soviétique naissante. L'ambition scientifique a pour but d'authentifier non pas les œuvres et les auteurs en eux-mêmes pour leur appartenance à une époque ou à un mouvement dont ils seraient particulièrement représentatifs, comme le remarque Davallon à propos des gestes de patrimonialisation (Davallon, 2006 : 122), mais plutôt d'opérer une relecture des œuvres dans le sens d'une histoire marxiste-léniniste de la littérature. Enfin, les chercheurs responsables de ce laboratoire sont également, pour partie, les enseignants des écrivains en formation, ce qui signale la continuité entre l'entreprise de patrimonialisation par l'archive et l'étude, et celle qui passe par l'enseignement et la production littéraire contemporaine.

Enfin, dans le cadre d'un institut territorialement aussi central que l'Institut Gorki, la littérature devient un moyen, parmi d'autres, d'unification de la jeune Union soviétique : les premières promotions de l'Institut semblent ainsi intégrer dans les bataillons des écrivains à venir des littérateurs issus de territoires périphériques (les «nations de l'Union des républiques socialistes soviétiques »).

Cette urgence dans la panthéonisation d'auteurs vivants peut s'expliquer par la courte existence de l'URSS et par ses ambitions sur la scène internationale. Dans ces premières années d'existence de l'Union, il est vital de démontrer, de façon plus ou moins forcée, la validité de l'idéologie marxiste-léniniste et, dans le cas de la littérature, d'établir rapidement un canon de grands auteurs et de grands textes pour doter l'URSS d'un « capital littéraire ».

\section{Le literaturokombinat : valeurs soviétiques et patrimoine littéraire}

On voit donc que le caractère massif et spontané des vocations littéraires dans l'Union soviétique des premières quinze années suscite un contre-mouvement de centralisation et d'institutionnalisation de la part du gouvernement, qui passe par l'élection d'une figure de guide en la personne de Gorki.

\section{Une institution totale : la production littéraire comme industrie, le patrimoine littéraire comme matière première}

Comme le remarque Katerina Clark (1978 : 193), la littérature est à cette époque réorganisée selon des principes appliqués ailleurs dans l'économie soviétique, et elle est considérée elle-même à terme comme une forme d'industrie. Dans les premiers mois d'existence du Litinstitut, ses fonctions se sont considérablement étendues. Pendant l'année 1932-1933, il est ainsi établi que l'institut à venir est un lieu qui rassemble toutes les étapes de la production littéraire, c'est-à-dire dans le même temps une fabrique de la littérature soviétique mais aussi un musée et un laboratoire.

Par rapport aux projets initiaux établis en 1932, le Litinstitut élargit son public dès 1933 : limité auparavant aux étudiants ayant déjà publié, l'enseignement de l'écriture y est désormais dispensé aux ouvriers et paysans n'ayant pas d'expérience littéraire. Enfin, l'institution se dote de sa propre maison d'édition, les Éditions Institut de littérature Gorki.

Parallèlement, le Litinstitut est conçu comme un musée de la littérature. Il est avant tout consacré à la personne de Gorki et doit comporter une bibliothèque, les archives personnelles de l'écrivain et un centre de recherche sur son œuvre. Le but est, selon une 
lettre que Gronski, le futur directeur, adresse à Gorki, de créer un " musée de la littérature communiste, ou de la littérature de la nouvelle ère socialiste, ou plus exactement un musée des initiateurs de la littérature de la nouvelle ère socialiste 4 ». Les reformulations successives de Gronski sont révélatrices de sa volonté de représenter, dans ce musée, le déroulement à venir de l'histoire littéraire, non seulement soviétique mais mondiale (« la nouvelle ère socialiste »). On peut ajouter à cela la dimension d'archive de la production soviétique, dans la mesure où l'Institut joue le rôle de bibliothèque de dépôt et doit recevoir un exemplaire de tous les livres littéraires produits en URSS.

Enfin, l'Institut Gorki doit être un laboratoire de la littérature, c'est-à-dire un lieu d'étude de l'histoire littéraire. Cette étude a pour but d'enrichir le patrimoine littéraire russe et de le mettre à la disposition des futurs écrivains que l'Institut forme par ailleurs. Comme le montrent les statuts de l'Institut cités plus haut, cela se concentre initialement sur les littératures nationales de l'ensemble de l'Union et sur les littératures contemporaines ; mais très rapidement, en lien avec l'ambition de former les nouveaux écrivains, la portée de cette recherche universitaire est étendue : un des premiers directeurs pressentis de l'Institut, Luppol, parle ainsi de rassembler autour de l'Institut " l'ensemble des forces vives de l'histoire littéraire » et évoque déjà en 1933 l'idée d'une histoire de la littérature mondiale en dix tomes (Kurilov, 2012 : 80). On prévoit déjà des chaires dans les différentes branches de la littérature mondiale (antique, française, allemande, anglo-américaine, italienne), de la littérature russe (ancienne, moderne et soviétique) et des littératures de l'Union (biélorusse, ukrainienne, géorgienne) ${ }^{5}$. Les plans quinquennaux qui suivent ajouteront à cela de nouvelles chaires : littératures romanes au sens plus large (portugaise, belge), littératures slaves, littératures scandinaves et littératures orientales. Luppol définit également les orientations de recherche de chaque chaire et met l'accent sur l'étude du réalisme (français, allemand et russe), désignant donc dans l'histoire littéraire ce qui doit inspirer la littérature soviétique. On doit aussi s'intéresser aux phénomènes qui s'inscrivent dans le récit marxiste-léniniste, par exemple à « l'influence de la révolution bourgeoise de 1789 sur la littérature mondiale ${ }^{6} »$.

On voit ici la dimension monumentale, multiple, exhaustive de l'Institut littéraire Gorki, mais également le lien explicite que son organisation entretient avec l'imaginaire soviétique. En effet, une lettre d'un des participants à sa fondation, Kamenev, évoque l'idée d'un « combinat », à la fois universitaire et pratique7. Dans cette image, il est clair que les écrivains en formation figurent le «produit final »- la littérature antérieure étant, elle, une forme de matière première, alors que la recherche, l'enseignement et les gestes de patrimonialisation qui les accompagnent (fondation d'un musée, d'une maison d'édition) relèvent du processus de transformation. La comparaison sert à rapprocher la littérature d'autres secteurs de production soviétiques " héroïques ", comme l'industrie et l'agriculture. La fameuse affirmation de Staline qui fait des écrivains des « ingénieurs des âmes » va dans le même sens.

\section{Faire de l'Union soviétique naissante le « méridien de Greenwich de la littérature »}

Comment est extraite cette matière première ? C'est l'objet du versant universitaire de l'Institut Gorki, et elle prend la forme de projets de recherche titanesques qui ne sont pas sans évoquer dans l'histoire littéraire internationale d'autres entreprises d'affirmation nationale d'une prééminence politique et culturelle.

28 Le premier projet de l'Institut Gorki porte sur la traduction ou la retraduction de classiques, avec l'ambition de produire des " éditions scientifiques commentées et solidement préfacées des monuments des littératures grecques et romaines ». De nombreux spécialistes des universités sont rassemblés pour ce projet (Kurilov, 2012 : 112).

Mais, surtout, la principale tâche savante de l'Institut Gorki est la rédaction d'une Histoire de la littérature universelle (Istoriâ vsemirnoj literatury), prévue dès la 
fondation de l'institution. Le terme russe choisi, vsemirnaâ (« de la totalité du monde »), avec la racine vse ( "tout »), et non mirovaâ (« mondiale »), n'est pas innocent. Il signale bien sûr l'ambition rétrospective totalisante de cette somme encyclopédique, mais il peut aussi être interprété dans un sens prospectif, par référence au Manifeste du parti communiste de Marx et Engels : l'arrivée du communisme devait en effet marquer la fin des littératures locales, nationales, au profit d'une littérature mondiale (la notion goethéenne, reprise par Marx, de Weltlitteratur en allemand, vsemirnaâ literatura dans les traductions en russe). Annoncée dans le premier plan quinquennal et prévue, avec optimisme, pour dix ou quinze ans plus tard, c'est en réalité une tâche qui accompagne toute la durée de vie soviétique de l'Institut et qui ne sera jamais achevée : les huit premiers tomes paraissent entre 1983 et 1994, et le dernier tome, qui devait justement porter sur l'histoire de la littérature de l'ère soviétique, ne paraitra jamais.

À en juger par l'introduction générale, publiée dans le premier tome en 1983, donc bien après la fondation de l'Institut, les principes de cette entreprise sont ceux d'une histoire conforme aux principes marxistes-léninistes : la littérature est le reflet des conditions socio-économiques, et, celles-ci évoluant vers le communisme, il est possible de dégager les lois (zakonomernost') de l'évolution littéraire dans tous les temps et dans tous les lieux. Toutefois, le but en est éminemment national, et il apparait bien vite qu'il s'agit moins de montrer le déroulement d'une histoire littéraire universelle que de démontrer le caractère universel de la littérature soviétique, voire, plus étroitement, russe, et de justifier son importance et l'attention qui lui est accordée au sein de l'Institut, aussi bien dans la conservation que dans l'enseignement. C'est ainsi qu'est présenté le tome 9, portant sur la littérature soviétique :
"Le concept de "contexte historique" est ici crucial. Il s'agit que le dévoilement de la logique interne de l'évolution des littératures nationales aille de pair avec la définition de la place qu'elles occupent à tel ou tel moment historique dans la dynamique commune de l'évolution de la littérature mondiale comprise comme macro-système. Seule l'application réussie d'une telle méthode peut permettre, par exemple, d'accomplir une des principales tâches que nous nous sommes données : dévoiler le sens international de la littérature russe. Cela ne peut se faire seulement par une approche qualitative, en analysant au cas par cas des exemples disparates, témoignant de l'influence de tel ou tel grand auteur russe sur les écrivains étrangers, mais avant tout au moyen d'une analyse objective des valeurs spirituelles développées dans la littérature russe et de la présentation des raisons historiques qui, aux xIxe-Xxe siècles, expliquent l'importance mondiale de ces valeurs » (Vipper, $1983: 9$ ).

On voit donc réapparaître ici la dimension nationale de cette histoire de la littérature mondiale. Ce discours patriotique s'inscrit également dans un retour du nationalisme en Russie soviétique postérieur à la fondation de l'Institut, mais il est cohérent avec l'ambition centralisatrice et totalisatrice des débuts de ce dernier.

Par conséquent, l'Institut Gorki, dans son versant universitaire, a pour but la réappropriation de l'ensemble du patrimoine littéraire mondial. Cette réappropriation permet à la littérature soviétique d'être inscrite dans le temps long et donc, paradoxalement, de prendre la tête de l'évolution littéraire moderne : selon Pascale Casanova (2008: 137), en effet :

\section{« La loi temporelle de l'univers littéraire peut s'énoncer ainsi : il faut être ancien pour avoir quelque chance d'être moderne ou de décréter la modernité. Il faut avoir un long passé national pour prétendre à l'existence littéraire pleinement reconnue dans le présent. »}

Certes le patrimoine littéraire strictement russe existe, mais dans cette somme d'histoire littéraire, il est insuffisant, et l'invention d'une nouvelle littérature soviétique s'accompagne d'une volonté de s'approprier et de résumer l'ensemble de la littérature mondiale. Ce processus est comparable à d'autres également analysés par Pascale Casanova : la traduction des classiques grecs et latins par les romantiques allemands au moment où l'unité allemande est en projet, ou encore l'importation des classiques grecs et latins par la Pléiade française dans le cadre de la translatio imperii et studii, quand 
les prétentions de la France de François Ier à la domination politique sur l'Europe, contre l'Italie, passe par l'affirmation d'une domination culturelle marquée par l'appropriation des littératures gréco-romaines par les écrivains français, contre les écrivains italiens.

Dans le cas de l'Institut Gorki, il s'agit à terme de démontrer que le processus historique littéraire mondial mène logiquement à la prééminence de la littérature soviétique et russe, et de justifier, par suite, la domination culturelle et donc politique de l'URSS. Au moyen d'une réinterprétation du patrimoine littéraire mondial et national, la littérature soviétique est amenée, selon la logique de cette nouvelle histoire littéraire marxiste-léniniste, à donner le tempo au monde littéraire. Pour emprunter encore à Pascale Casanova (2008 : 135), l'Institut Gorki et son travail patrimoine visent à faire de Moscou le nouveau « méridien de Greenwich de la littérature ».

\section{L'Institut Gorki après 1991 : postérité et réinterprétation du patrimoine littéraire}

Cette fonction de gardien du patrimoine persiste aujourd'hui dans le Litinstitut, qui reste une université publique formant en cinq ans des "travailleurs de la culture » (mention figurant sur les diplômes actuels des étudiantes et étudiants de l'Institut) : pour en mesurer l'importance, il faudrait faire toute l'histoire de l'Institut et de ses pratiques pédagogiques et universitaires. On peut au moins s'attacher à une autre production monumentale du Litinstitut, en plusieurs tomes, parue en $2013:$ Souvenirs de l'Institut littéraire, dont le titre signale la dimension mémorielle. Il s'agit de quatre tomes d'extraits de journaux, de mémoires, de discours, d'extraits d'œuvres des élèves, anciens élèves et professeurs du Litinstitut. Les deux derniers tomes sont particulièrement révélateurs du projet actuel du Litinstitut.

36 Le dernier se clôt sur une postface de Boris Tarasov, recteur de l'Institut à l'époque de la publication, qui affirme le rôle que doit jouer le Litinstitut dans le contexte actuel post-soviétique. Il insiste sur la continuité de l'Institut actuel avec celui de la période soviétique ; tout en reconnaissant la dureté de la censure soviétique, il vante la création d'un milieu professionnel remarquable et affirme que l'Institut est appelé à jouer le même rôle maintenant, avec une note toutefois plus conservatrice :

\footnotetext{
« Aujourd'hui, voilà venus d'autres temps où, dans le cadre d'un pragmatisme total et d'une mercantilisation de la vie qui, par-là, perd son sens, s'élaborent de nouveaux diktats et de nouveaux obstacles idéologiques, plus subtils, moins visibles, mais plus forts et plus efficaces, de nouveaux mécanismes de pression sur la liberté personnelle, des renversements des vraies valeurs, des travestissements de la valeur réelle de la création, y compris littéraire. La poésie authentique, si elle n'est pas ironique ou satirique, recule au second plan. Règnent la fantasy, les romans policiers, les romans d'aventures, les romans féminins et autres, ainsi qu'une littérature de masse qui s'est infiltrée dans la conscience de l'écrivain par le moyen d'une propagande publicitaire, d'adaptations télévisées, de projets éditoriaux destinés à dégager du profit 8 » (Tarasov, 2013 :508).
}

En réponse à cela, Tarasov (ibid. : 509) insiste sur le fait que l'Institut, dans la formation qu'il donne aux écrivains à venir, doit défendre le patrimoine littéraire russe contre ces formes nouvelles et renforcer les critères d'un art verbal authentique, raviver et développer, dans une situation historique et littéraire changée et changeante, les meilleures traditions, dont celles du Litinstitut, qui permettront de distinguer et d'évaluer les phénomènes véritablement significatifs dans le chaos de la littérature contemporaine ${ }^{9}$.

Le caractère conservateur de ces déclarations montre un changement dans le régime d'historicité, d'une conception de l'histoire tournée vers le futur dans les premiers temps de l'URSS à un passéisme de plus en plus marqué dès avant la chute de l'Union, et surtout sous les mandats successifs de Vladimir Poutine. Toutefois, même si les principes idéologiques ont changé, on voit que l'Institut conserve sa fonction d'identification et de valorisation des phénomènes " significatifs », potentiellement 
canoniques, dans la littérature contemporaine : il doit être le gardien du patrimoine littéraire vivant russe. C'est ce que montre également le poème qui ouvre le troisième tome des Souvenirs, "Litinstitut », du professeur Vladimir Kostrov, qui dirige les séminaires de poésie depuis 1979. Il s'agit d'un texte en tétramètre classique, qui décrit le bâtiment principal de l'Institut, la maison Herzen, et dont je traduis la fin :

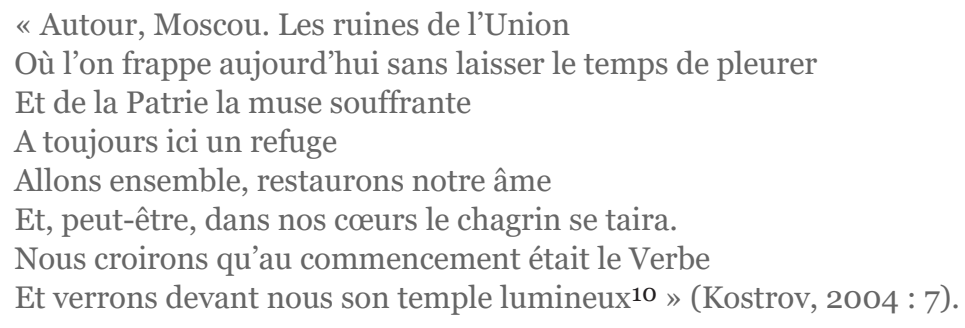

$\mathrm{Au}$ lien entre nation et littérature, fondé du temps de l'URSS sur l'idéologie marxiste prolétarienne, s'ajoute, sous le régime poutinien, un troisième terme, celui de la religion : le « Verbe », aussi bien divin que littéraire, fait de la Russie (et du Litinstitut) la gardienne de l'idée sacrée de littérature, qui est, par essence, nationale.

En conclusion, on peut affirmer que, pendant la période soviétique de l'existence de l'Institut littéraire Gorki, une importance croissante est accordée à l'histoire littéraire, d'abord nationale, puis mondiale. Son étude est soumise avant tout à un but pédagogique de formation des écrivains, puis devient une tâche autonome et centrale de l'Institut.

41 Cette place prise par l'histoire littéraire aboutit à une patrimonialisation de la littérature. Cela est visible premièrement dans le caractère monumental du combinat littéraire, dans les projets titanesques dont il se charge, et se manifeste symboliquement par son installation dans la maison Herzen, haut lieu de la vie littéraire russe du XIXe siècle : la patrimonialisation par avance de la littérature soviétique passe aussi par son installation dans un symbole du patrimoine littéraire russe, même s'il s'agit du patrimoine de la Russie tsariste, approprié et remotivé par le nouveau pouvoir. Cet accent mis sur la littérature passée dans la formation des écrivains futurs signifie qu’il s'agit de produire une littérature certes nouvelle, une littérature du futur, mais qui, en un sens, soit déjà canonique. En témoigne le nom même de l'Institut : honorer un écrivain vivant, le déclarer patrimoine national en lui donnant sa bibliothèque et ses archives implique qu'il n'y a pas seulement valorisation d'un patrimoine littéraire passé, mais aussi patrimonialisation d'une littérature contemporaine déjà devenue classique.

On peut faire l'hypothèse à partir de ces dernières remarques que le rôle de gardien de la culture que joue le Litinstitut, toujours institution publique aujourd'hui, n'est pas seulement le résultat d'un tournant conservateur ou d'un retour aux sources nationales lié à la fin de l'URSS et à l'avènement de Poutine. Il est au contraire dans la continuité du projet soviétique : la prise en charge de l'enseignement de l'écriture littéraire par des instituts centralisés et gouvernementaux s'explique ici par un lien encore très fort entre l'idée de nation, l'idée d'histoire et l'idée de littérature, ce qui explique la présence très forte du patrimoine littéraire dans ces lieux de création. Il paraîtrait donc logique qu'un État centralisé, fort, autoritaire (soviétique ou post-soviétique), s'empare de l'enseignement de la création littéraire afin à la fois de modeler l'histoire littéraire des années à venir et d'affirmer sa pérennité via la valorisation du patrimoine littéraire du passé.

\section{Bibliographie}

Casanova (Pascale). 2008. La République mondiale des lettres [1999]. Paris : Seuil (Points essais).

Clark (Katerina). 1978. " Little heroes and big deeds: literature responds to the first five-year plan », p. 189-206 in Cultural Revolution in Russia. 1928-1931 / sous la direction de Sheila Fitzpatrick. Bloomington : Indiana University Press.

Davallon (Jean). 2006. Le Don du patrimoine : Une approche communicationnelle de la patrimonialisation. Paris : Hermes Science - Lavoisier (Communication, médiation et construits 
sociaux).

Deschepper (Julie). 2018. "Le "patrimoine soviétique" de l'URSS à la Russie contemporaine. Généalogie d'un concept ». Vingtième Siècle, 137, p. 77-98.

Dobrenko (Evgenij). 1999. Formovka sovietskogo pisatelâ [La Création de l'écrivain soviétique]. Saint-Pétersbourg : Akademičeskij Proekt.

Günther (Hans). 1993. Der sozialistische Übermensch. Maksim Gor'kij und die sowjetische Heldenmythos. Stuttgart, Weimar : Verlag J. B. Metzler.

Kostrov (Vladimir). 2004. "Litinstitut » [Le Litinstitut], p. 7 in Vospominaniâ o Literaturnom institute. Kniga tret'â [Souvenirs de l'Institut littéraire. Livre III]. Moscou : Izdatel'stvo Literaturnogo instituta im. A. M. Gor'kogo.

Kurilov (Aleksandr). 2011. " Gor'kij i Večernij rabočij literaturnyj universitet (VRLU) » [Gorki et l’Université ouvrière littéraire du soir (VRLU)]. Literaturovedčeskij Žurnal, 29, p. 259-281.

Kurilov (Aleksandr). 2012. « Iz istorii Instituta: A.M. Gor'kij i IMLI; Pervye položeniâ ob Institute i plany naučnoj raboty » [Extraits de l'histoire de l'Institut : Gorki et l'IMLI, premiers règlements et plans de travail universitaire]. Literaturovedčeskij Žurnal, 31, p. 72-139.

Kurilov (Aleksandr). 2015. " Kak sozdavalsâ litinstitut » [Comment le Litinstitut a été fondé]. Site Literaturnâ̂ Rossiâ, mis en ligne le 23 février 2015, $\mathrm{n}^{\circ}$ 2008/52 : https://litrossia.ru/item/3242-oldarchive/ [consulté le 9 août 2021].

Poulot (Dominique). 2006. Une histoire du patrimoine en Occident, XVIII-XXIe siècle. Paris : PUF (Le nœud gordien).

Tarasov (Boris). 2013. « Dom Gercena. Na Tverskom bul'vare, 25 » [La maison Herzen. Au 25, boulevard Tverskoï], p. 508-512 in Vospominaniâ o Literaturnom institute. Kniga četvertaâ [Souvenirs de l'Institut littéraire. Livre IV]. Moscou : Izdatel'stvo Literaturnogo instituta im. A. M. Gor'kogo.

Timasheff (Nicholas). 1946. The Great Retreat. New York : Dutton.

Unfried (Berthold). 1999. " Montée et déclin des héros », p. 189-202 in La Fabrique des héros / sous la direction de Pierre Centlivres, Daniel Fabre et Françoise Zonabend. Paris : Éditions de la Maison des sciences de l'homme (Cahiers d'ethnologie de la France, 12), en ligne : https://books.openedition.org/editionsmsh/4029?lang=fr [consulté le 9 août 2021].

Vipper (Ûrij). 1983. "Vstupitel'nye zamečaniâ » [Remarques introductives], p. 5-12 in Istoriâ vsemirnoj literatury / sous la direction de G. P. Berdnikov. Moscou : Nauka.

\section{Notes}

1 On trouve dans la jeune littérature soviétique des traces de ce foisonnement littéraire. Par exemple, l'écrivain Mikhail Zochtchenko publie en 1929 un recueil de Nouvelles sentimentales qu'il attribue à un certain Kolenkorov, rencontré dans un club littéraire ouvrier et dont il aurait amendé la prose avant de la publier. En réalité pastiches de la jeune littérature soviétique, ces textes tracent en filigrane le portrait d'un écrivain médiocre, proche de la figure du graphomane omniprésente dans la littérature russe prérévolutionnaire, et que l'on tente de guérir, avec plus ou moins de succès, de son esthétique petite-bourgeoise. Une des préfaces aux Nouvelles sentimentales, écrite par Zochtchenko, explique ainsi que l'écrivain Kolenkorov, qui faisait partie de l'aile droite des compagnons de route de la révolution, est en train de se réformer sous son influence, et sous celle du club littéraire ouvrier dont il fait partie, et deviendra un représentant notable de « l'école naturaliste ». La critique est ici à double tranchant : elle porte à la fois, conformément à l'idéologie soviétique, sur la littérature " petite-bourgeoise » et, en même temps, elle raille le conformisme grandissant des écrivains d'URSS et la médiocrité de cette littérature des temps nouveaux, dont le ridicule Kolenkorov a si peu de mal à devenir un chef de file.

2 Nous traduisons.

3 Pravda, 26 septembre 1932, p. 1. Nous traduisons.

4 Archives Gorki. Nous traduisons.

5 Archives Gorki.

6 Archives de l'Académie des sciences de Russie.

7 Literatournaïa Gazeta, 26 juillet 1934, p. 1.

8 Nous traduisons.

9 Nous traduisons.

10 Nous traduisons. 
Référence papier

Sylvia Chassaing, « L'Institut littéraire Gorki à Moscou : la patrimonialisation de la création littéraire », Culture \& Musées, 38 | 2021, 51-68.

Référence électronique

Sylvia Chassaing, "L'Institut littéraire Gorki à Moscou : la patrimonialisation de la création littéraire », Culture \& Musées [En ligne], 38 | 2021, mis en ligne le 10 novembre 2021, consulté le 08 décembre 2021. URL : http://journals.openedition.org/culturemusees/6673 ; DOI :

https://doi.org/10.4000/culturemusees.6673

\section{Auteur}

\section{Sylvia Chassaing}

Université Paris 8, Centre d'études franco-russe de Moscou

Sylvia Chassaing est docteure en littérature comparée de l'Université Paris 8 et travaille sur les rapports entre la production littéraire et artistique et les institutions qui l'encadrent dans les domaines français, états-unien et russe. Elle a codirigé avec Cyril Barde et Hermeline Pernoud la journée d'étude et la publication de l'ouvrage Fin-de-siècle : fin de l'art ? Destins de l'art dans les discours de la fin des XIXe et XXe siècles (Presses Sorbonne nouvelle, 2018). Elle est également l'autrice d'articles sur les formes de contestation de l'autorité politique dans les domaines littéraire et artistique, spécifiquement dans les aires linguistiques russes et polonaises : «Tret'a volna akcionizma: iskusstvo svobodnogo dejstvija vo vremja reakcji » [La troisième vague de l'actionnisme : l'art de l'acte libre en période réactionnaire] (avec Pavel Mitenko, Moskovskij Khudozestvennyj Zurnal, $\left.n^{\circ} 102,2017\right)$, « Manifester en silence ? \#Tikhiypiket ou l'activisme littéraire à l'heure des réseaux sociaux » (Essais, $\mathrm{n}^{\circ}$ 16, 2020) et " Robots poètes chez Stanisław Lem : réflexions sur la nécessaire étrangeté de la langue littéraire en contexte soviétique »(ReS Futurae, $\left.\mathrm{n}^{\circ} 16,2020\right)$

Courriel : sylvia.chassaing[at]gmail.com

\section{Droits d'auteur}

Culture \& Musées 